Article

\title{
An Optimal Pursuit Differential Game Problem with One Evader and Many Pursuers
}

\author{
Idris Ahmed 1,2,3 (D), Poom Kumam 1,2,* (D), Gafurjan Ibragimov ${ }^{4}$ (D) Jewaidu Rilwan ${ }^{1,2,5}$ (D) and \\ Wiyada Kumam ${ }^{6, *}$ (D)
}

1 KMUTTFixed Point Research Laboratory, Room SCL 802 Fixed Point Laboratory, KMUTT-Fixed Point Theory and Applications Research Group (KMUTT-FPTA), Science Laboratory Building, Department of Mathematics, Faculty of Science, King Mongkut's University of Technology Thonburi (KMUTT), 126 Pracha-Uthit Road, Bang Mod, Thrung Khru, Bangkok 10140, Thailand

2 Center of Excellence in Theoretical and Computational Science (TaCS-CoE), Science Laboratory Building, Faculty of Science, King Mongkut's University of Technology Thonburi (KMUTT), 126 Pracha-Uthit Road, Bang Mod, Thrung Khru, Bangkok 10140, Thailand

3 Department of Mathematics and Computer Science, Sule Lamido University, P.M.B 048 Kafin-Hausa, Jigawa State, Nigeria

4 Institute for Mathematical Research and Department of Mathematics, Faculty of Science (FS), Universiti Putra Malaysia, Selangor, Serdang 43400, Malaysia

5 Department of Mathematical Sciences, Faculty of Physical Sciences, Bayero University, P.M.B 3011 Kano, Nigeria

6 Program in Applied Statistics, Department of Mathematics and Computer Science, Faculty of Science and Technology, Rajamangala University of Technology Thanyaburi (RMUTT), Thanyaburi, Pathumthani 12110, Thailand

* Correspondence: poom.kum@kmutt.ac.th (P.K.); wiyada.kum@rmutt.ac.th (W.K.)

Received: 1 August 2019; Accepted: 2 September 2019; Published: 11 September 2019

\begin{abstract}
The objective of this paper is to study a pursuit differential game with finite or countably number of pursuers and one evader. The game is described by differential equations in $l_{2}$-space, and integral constraints are imposed on the control function of the players. The duration of the game is fixed and the payoff functional is the greatest lower bound of distances between the pursuers and evader when the game is terminated. However, we discuss the condition for finding the value of the game and construct the optimal strategies of the players which ensure the completion of the game. An important fact to note is that we relaxed the usual conditions on the energy resources of the players. Finally, some examples are provided to illustrate our result.
\end{abstract}

Keywords: pursuit; control functions; integral constraints; strategies; value of the game

\section{Introduction}

The differential game has been an area of great interest to many applied mathematicians due to its application in solving real life problems in knowledge areas such as economics, engineering, missile guidance, behavioral biology. The first to study differential game was Rufus Isaacs [1], and one of the games analyzed was "the homicidal chauffeur game". For the fundamental concepts of differential games, see [1-8].

There are many different types of differential game problems, and one type is called pursuit-evasion differential game. Pursuit-evasion differential game is a game involving two players, called pursuer and evader, with conflicting goals. The aim of the pursuer is to complete the game in a finite time, whereas that of the evader is contrary. Strategies of pursuit and evasion play a role in many areas of life, such as missile launched at enemy aircraft, coastguard saving shipwrecked 
sailors, etc. The problem of constructing optimal strategies and finding the value of the game in a pursuit-evasion differential game motivated a lot of researchers to study this class of differential game problems, and fundamental results have been obtained, see [9-13].

Differential games of many pursuers with the integral and geometry constrained were studied by $[9,14-19]$. The case where the state variables are constraints was studied in [20], they considered a nonempty closed convex set in a plane, with the pursuers and evader movement restricted within the set during the game. Conditions under which pursuit could be completed were obtained and the strategies for the pursuers were constructed.

The evasion differential game of two dimensions, which involves one evader and several pursuers, was studied in [11]. The control functions of the players were subject with integral constraints. The game is solved by presenting explicit strategy for the evader, which guarantees evasion under the condition that there is no relation between the energy resource of the players.

In [21] Levchenko and Pashkov considered differential games described by simple differential equations, where the controls obeyed integral constraints. However, they showed that irrespective of the resources for controls of an individual, the completion of the game remains doable.

Ibragimov and Satimov [22] obtained sufficient condition for the completion of pursuit in a differential game problem of several pursuers and evaders in the space $\mathbb{R}^{n}$, with integral constraints on the control functions of the players. The results were obtained under the condition that the energy resource of the pursuers is greater than that of the evaders.

Ibragimov [9] studied a differential game of a countable number of pursuers pursuing one evader in Hilbert space $l_{2}$, with geometric constraints on the control functions of the pursuers and evader. Optimal strategies of the players were constructed and optimal pursuit time was found, under the assumption that the energy resource of the pursuers is greater than that of the evader.

Ibragimov and Kuchkarov [10] considered the same problem in [9], with integral constraints imposed on the control functions of the players. In this case, optimal strategies were constructed and value of the game was found under the assumption that energy resource of the evader is greater than that of any pursuers.

Salimi and Ferrara [12] studied a simple motion differential game with finite number of pursuers and one evader with integral constraints imposed on control of the players in Hilbert space $l_{2}$. The equations of motion are described by

$$
\begin{gathered}
P_{i}: \dot{x}_{i}(t)=u_{i}(t), \quad x_{i}(0)=x_{i 0} \\
E: \dot{y}(t)=v(t), \quad y(0)=y_{0}
\end{gathered}
$$

where $u_{i}$ is the control function of the $i^{t h}$ pursuers and $v$ is that of the evader. The authors solved the problem and found the value of the game under the assumption that the energy resource of each pursuer is not necessarily greater than that of the evader, and optimal strategies of the pursuers were also constructed.

Inspired by the results in [9-12,22] and some known results on optimal pursuit problem in a Hilbert space $l_{2}$, the objective of this paper is to construct the optimal strategies and finding value of the game such that there is no relation between the energy resource of the players.

This paper is sectioned as follows. The second section present statement of the problems and some useful definitions which will be required for the later sections. In the third section, attainability domain of the players, optimal strategies and value of the game and some examples are given to show the application of the obtained results. In the last section, we give the concluding part of the paper.

\section{Statement of the Problem}

In this section, we present the statement of the problem and some useful definitions that will be used to prove our main theorem. 
Here, we will consider an optimal pursuit problem with finite or countably many pursuers and one evader in a Hilbert space $l_{2}$, in such away that there is no relation between the their energy resources. In the space $l_{2}$, with elements

$$
\alpha=\left(\alpha_{1}, \ldots, \alpha_{k}, \ldots\right), \quad \sum_{k=1}^{\infty} \alpha_{k}^{2}<\infty,
$$

the inner product and norm are defined as

$$
(\alpha, \beta)=\sum_{k=1}^{\infty} \alpha_{k} \beta_{k}, \quad\|\alpha\|=\left(\sum_{k=1}^{\infty} \alpha_{k}^{2}\right)^{1 / 2} .
$$

Let $P_{i}$ and $\mathrm{E}$ denote the motions of the pursuers and the evader, whose equations are described by

$$
\begin{aligned}
P_{i}: \dot{x}_{i}(t) & =\eta(t) u_{i}(t), \quad x_{i}(0)=x_{i 0}, \\
E: \dot{y}(t) & =\eta(t) v(t), \quad y(0)=y_{0},
\end{aligned}
$$

where $x_{i}(t), x_{i 0}, u_{i}(t), y(t), y_{0}, v(t) \in l_{2}, u_{i}=\left(u_{i 1}, \ldots, u_{i k}, \ldots\right)$ and $v=\left(v_{1}, \ldots, v_{k}, \ldots\right)$ are the control parameters of pursuer $P_{i}$ and evader E, respectively. Throughout this paper, $i \in I=\{1,2,3, \ldots m\}$.

Let $\theta$ be a fixed time, and the function $\eta(t)$ be nonzero on any open interval-scalar measurable and square integrable over the interval $[0, \tau], \tau>0$. It is also assumed to satisfy the following conditions:

$$
\begin{gathered}
a(\tau)=\left(\int_{0}^{\tau} \eta^{2}(t) d t\right)^{1 / 2}<\infty . \\
H\left(x_{0}, r\right)=\left\{x \in l_{2}:\left\|x-x_{0}\right\| \leq r\right\}, \quad S\left(x_{0}, r\right)=\left\{x \in l_{2}:\left\|x-x_{0}\right\|=r\right\},
\end{gathered}
$$

denote the ball and sphere, respectively, in the space $l_{2}$ with center $x_{0}$ and radius $r$. We now give some useful definitions.

Definition 1. The admissible control of the ith pursuer is a function $u(\cdot), u_{i}:[0, \theta] \rightarrow l_{2}$ defined as

$$
\left\|u_{i}(\cdot)\right\|_{2}=\left(\int_{0}^{\theta}\left\|u_{i}(s)\right\|^{2} d s\right)^{1 / 2} \leq \rho_{i}, \quad\left\|u_{i}\right\|=\left(\sum_{k=1}^{\infty} u_{i k}^{2}\right)^{1 / 2},
$$

provided that $u_{i k}:[0, \theta] \rightarrow \mathbb{R}^{1}, k=1,2, \ldots$, are Borel measurable functions and $\rho_{i}$ is a fixed positive number $\forall i$. Let $B\left(\rho_{i}\right)$ denote the set of all admissible controls of the pursuer $x_{i}$.

Definition 2. The admissible control of the evader is a function $v(\cdot), v:[0, \theta] \rightarrow l_{2}$ defined as

$$
\|v(\cdot)\|_{2}=\left(\int_{0}^{\theta}\|v(s)\|^{2} d s\right)^{1 / 2} \leq \sigma,
$$

where $v_{k}:[0, \theta] \rightarrow \mathbb{R}^{1}, k=1,2, \ldots$, are Borel measurable functions and $\sigma$ is a fixed positive number. Let $B(\sigma)$. denote the set of all admissible controls of the evader $y$.

Once the players admissible controls $u_{i}(\cdot) \in B\left(\rho_{i}\right)$ and $v(\cdot) \in B(\sigma)$ are chosen, the corresponding motion $x_{i}(\cdot)$ and $y(\cdot)$ of the players are defined as

$$
\begin{gathered}
x_{i}(t)=\left(x_{i 1}(t), x_{i 2}(t), \ldots, x_{i k}(t), \ldots\right), \quad y(t)=\left(y_{1}(t), y_{2}(t), \ldots, y_{k}(t), \ldots\right), \\
x_{i k}(t)=x_{i k 0}+\int_{0}^{\theta} \eta(s) u_{i k}(s) d s, \quad y_{k}(t)=y_{k 0}+\int_{0}^{\theta} \eta(s) v_{k}(s) d s, \quad i \in I, \quad k \in N .
\end{gathered}
$$


It is not difficult to see that $x_{i}(\cdot), y(\cdot) \in C\left(0, \theta ; l_{2}\right)$, where $C\left(0, \theta ; l_{2}\right)$ is the space of functions

$$
f(t)=\left(f_{1}(t), f_{2}(t), \ldots,\right) \in l_{2}, \quad t \geq 0,
$$

such that

(i) $f_{k}(t), 0 \leq t \leq \theta, k \in N$, are absolutely continuous functions;

(ii) $f(t), 0 \leq t \leq \theta$, is continuous with respect to the norm on $l_{2}$ space.

Definition 3. The strategy of the pursuer $P_{i}$ is a function $U_{i}\left(t, x_{i}, y, v\right) . U_{i}:[0, \infty) \times l_{2} \times l_{2} \times l_{2} \rightarrow l_{2}$, such that the system of equations

$$
\begin{aligned}
\dot{x}_{i}(t) & =\eta(t) U_{i}\left(t, x_{i}, y, v(t)\right), \quad x_{i}(0)=x_{i 0}, \\
\dot{y}(t) & =\eta(t) v(t), \quad y(0)=y_{0},
\end{aligned}
$$

has a unique solution $\left(x_{i}(\cdot), y(\cdot)\right)$ for any $x_{i}(\cdot), y(\cdot) \in C\left(0, \theta ; l_{2}\right)$ and admissible control $v=v(t), 0 \leq t \leq \theta$, of the evader $E$. We said that the strategy $U_{i}$ is admissible if each control formed by strategy $U_{i}$ is admissible.

Definition 4. The optimal strategies $U_{i 0}$ of the pursuers $P_{i}$ are defined as

$$
\inf _{U_{1}, \ldots, U_{m}, \ldots} \Gamma_{1}\left(U_{1}, \ldots, U_{m}, \ldots\right)=\Gamma_{1}\left(U_{10}, \ldots, U_{m 0}, \ldots\right)
$$

such that

$$
\Gamma_{1}\left(U_{1}, \ldots, U_{m}, \ldots\right)=\sup _{v(\cdot)} \inf _{i \in I}\left\|x_{i 0}-y(\theta)\right\|
$$

where $U_{i}$ and $v(\cdot)$ are admissible strategies of the pursuers $P_{i}$ and evader $E$, respectively.

Definition 5. The strategy of evader $E$ is a function $V\left(t, x_{1}, \ldots, x_{m}, \ldots, y\right), V:[0, \infty) \times l_{2} \times \cdots \times l_{2} \times$ $\cdots \times l_{2} \rightarrow l_{2}$, such that the system of equations

$$
\begin{aligned}
\dot{x}_{i}(t) & =\eta(t) u_{i}\left(t, x_{i}, y, v(t)\right), \quad x_{i}(0)=x_{i 0}, \\
\dot{y}(t) & =\eta(t) V\left(t, x_{1}, \ldots, x_{m}, \ldots, y_{1}\right), \quad y(0)=y_{0},
\end{aligned}
$$

has a unique solution $\left(x_{i}(\cdot), \ldots, x_{m}(\cdot), \ldots y(\cdot)\right)$ for any $x_{i}(\cdot), y(\cdot) \in C\left(0, \theta ; l_{2}\right)$, and admissible controls $u_{i}=u_{i}(t), 0 \leq t \leq \theta$ of the pursuers $P_{i}$. We said that strategy $V$ is admissible if each control formed by strategy $V$ is admissible.

Definition 6. The optimal strategy $V_{0}$ of the evader $E$ is defined as

$$
\sup _{V} \Gamma_{2}(V)=\Gamma_{2}\left(V_{0}\right)
$$

provided that

$$
\Gamma_{2}(V)=\inf _{u_{1}(\cdot), \ldots, u_{m}(\cdot), \ldots} \inf _{i \in I}\left\|x_{i 0}-y(\theta)\right\|,
$$

$u_{i}$ are admissible control of the pursuers $P_{i}$ and $V$ is that of the evader $E$.

If $\Gamma_{1}\left(U_{10}, \ldots, U_{m 0}, \ldots\right)=\Gamma_{2}\left(V_{0}\right)=\lambda$, then, problem (2) has a value $\lambda$.

Our aim is to find the optimal strategies $U_{i 0}, V_{0}$ of the players and value of the game, respectively. 


\section{Auxiliary Game}

It is easily to see that the attainability domain of the pursuer $P_{i}$ from the initial position $x_{i 0}$ at time $\theta$ is the closed ball $H\left(x_{i 0}, a(\theta) \rho_{i}\right)$. Indeed

$$
\begin{array}{r}
\left\|x(\theta)-x_{i 0}\right\|=\left\|\int_{0}^{\theta} \eta(s) u_{i}(s) d s\right\| \leq \int_{0}^{\theta} \eta(s)\left\|u_{i}(s)\right\| d s \\
\leq\left(\int_{0}^{\theta} \eta^{2}(s) d s \int_{0}^{\theta}\left\|u_{i}(s)\right\|^{2} d s\right)^{1 / 2} \leq a(\theta) \rho_{i} .
\end{array}
$$

Moreover, if $\bar{x} \in H\left(x_{i 0}, a(\theta) \rho_{i}\right)$, that is, $\left\|\bar{x}-x_{i 0}\right\| \leq a(\theta) \rho_{i}$, then, for the control

$$
u_{i}(s)=\frac{\eta(s)}{a^{2}(\theta)}\left(\bar{x}-x_{i 0}\right), \quad 0 \leq s \leq \theta
$$

of the pursuer, we get

$$
\begin{aligned}
x_{i}(\theta) & =x_{i 0}+\int_{0}^{\theta} \eta(s) u_{i}(s) d s \\
& =x_{i 0}+\int_{0}^{\theta} \eta(s)\left(\frac{\eta(s)}{a^{2}(\theta)}\left(\bar{x}-x_{i 0}\right)\right) d s \\
& =x_{i 0}+\frac{\bar{x}-x_{i 0}}{a^{2}(\theta)} \int_{0}^{\theta} \eta^{2}(s) d s \\
& =\bar{x} .
\end{aligned}
$$

Therefore, the admissibility of this control follows from the relation

$$
\int_{0}^{\theta}\left\|u_{i}(s)\right\|^{2} d s=\int_{0}^{\theta}\left\|\frac{\eta(s)}{a^{2}(\theta)}\left(\bar{x}-x_{i 0}\right)\right\|^{2} d s \leq \frac{\left\|\bar{x}-x_{i 0}\right\|^{2}}{a^{4}(\theta)} a^{2}(\theta) \leq \rho_{i}^{2} .
$$

Moreover, applying the same procedure one can see that the attainability domain of the evader $E$ at time $\theta$ from the initial state $y_{0}$ is the ball $H\left(y_{0}, a(\theta) \sigma\right)$.

In this section, we study a differential game of one pursuer $x$ and one evader $y$. For simplicity, we use the notation $\rho_{i}=\rho, x_{i 0}=x_{0}$, and $x_{i}=x$. Then, dynamics of $x$ and $y$ are described by

$$
\begin{array}{ll}
P: \dot{x}=\eta(t) u, & x(0)=x_{0}, \\
E: \dot{y}=\eta(t) v, & y(0)=y_{0} .
\end{array}
$$

The target of the pursuer $P$ is to perceive the equality $x(\tau)=y(\tau)$ at some $\tau, 0 \leq \tau \leq \theta$; and that of the evader $E$ is contrary.

Let

$$
X=\left\{z: 2\left(y_{0}-x_{0}, z\right) \leq a^{2}(\theta)\left(\rho^{2}-\sigma^{2}\right)+\left\|y_{0}\right\|^{2}-\left\|x_{0}\right\|^{2}\right\}
$$

Lemma 1. If $y(\theta) \in X$, then, there exists an admissible strategy of the pursuer $P$ which ensures $x(\theta)=y(\theta)$ in the game (4).

Proof. Suppose the assumption of Lemma 1 holds, construct the strategy of the pursuer as follows:

$$
u(t)=\frac{\eta(t)}{a^{2}(\theta)}\left(y_{0}-x_{0}\right)+v(t), \quad 0 \leq t \leq \theta .
$$

Then, admissibility of this strategy can be proved as follows. Since

$$
y(\theta)=y_{0}+\int_{0}^{\theta} \eta(s) v(s) d s \in X
$$


then,

$$
\begin{aligned}
2\left(y_{0}-x_{0}, y(\theta)\right) & \leq a^{2}(\theta)\left(\rho^{2}-\sigma^{2}\right)+\left\|y_{0}\right\|^{2}-\left\|x_{0}\right\|^{2}, \\
2\left(y_{0}-x_{0}, y_{0}+\int_{0}^{\theta} \eta(t) v(t) d t\right) & \leq a^{2}(\theta)\left(\rho^{2}-\sigma^{2} \theta\right)+\left\|y_{0}\right\|^{2}-\left\|x_{0}\right\|^{2}, \\
2\left(y_{0}-x_{0}, \int_{0}^{\theta} \eta(t) v(t)\right) & \leq a^{2}(\theta)\left(\rho^{2}-\sigma^{2}\right)-\left(\left\|y_{0}\right\|^{2}-2\left(x_{0}, y_{0}\right)+\left\|x_{0}\right\|^{2}\right) .
\end{aligned}
$$

Hence from the strategy (5) and inequality (6), we have

$$
\begin{aligned}
\int_{0}^{\theta}\|u(t)\|^{2} d t & =\int_{0}^{\theta}\left\|\left(\frac{\eta(t)}{a^{2}(\theta)}\left(y_{0}-x_{0}\right)+v(t)\right)\right\|^{2} d t \\
& =\int_{0}^{\theta} \frac{\eta^{2}(t)}{a^{4}(\theta)}\left\|y_{0}-x_{0}\right\|^{2} d t+\frac{2}{a^{2}(\theta)} \int_{0}^{\theta} \eta(t)\left(y_{0}-x_{0}, v(t)\right) d t+\int_{0}^{\theta}\|v(t)\|^{2} d t \\
& \leq \frac{\left\|y_{0}-x_{0}\right\|^{2}}{a^{4}(\theta)} a^{2}(\theta)+\frac{1}{a^{2}(\theta)}\left(a^{2}(\theta)\left(\rho^{2}-\sigma^{2}\right)-\left\|y_{0}-x_{0}\right\|^{2}\right)+\sigma^{2} \\
& =\rho^{2} .
\end{aligned}
$$

This shows that the strategy (5) is admissible. Therefore,

$$
\begin{aligned}
x(\theta) & =x_{0}+\int_{0}^{\theta} \eta(t)\left(\frac{\eta(t)}{a^{2}(\theta)}\left(y_{0}-x_{0}\right)+v(t)\right) d t \\
& =x_{0}+\frac{\left(y_{0}-x_{0}\right)}{a^{2}(\theta)} \int_{0}^{\theta} \eta^{2}(t) d t+\int_{0}^{\theta} \eta(t) v(t) d t \\
& =x_{0}+y_{0}-x_{0}+\int_{0}^{\theta} \eta(t) v(t) d t \\
& =y(\theta) .
\end{aligned}
$$

This proves the lemma.

Remark 1. It should be noted that in the construction of the pursuer's strategy we do not require the inequality $\rho \geq \sigma$.

\section{Main Result}

We recall the following lemmas in order to prove our main theorem.

Lemma 2. (see Ibragimov et al. 2005. Lemma 9). Suppose $r$ and $R_{i}, i \in I$, are fixed positive real numbers and $H\left(x_{i 0}, R_{i}\right), i \in I$, and $H\left(y_{0}, r\right)$ are collections of finitely or a countable number of closed balls. Let

$$
\begin{aligned}
I_{0} & =\left\{i \in I: S\left(y_{0}, r\right) \cap H\left(x_{i 0}, R_{i}\right) \neq \varnothing\right\}, \\
X_{i} & =\left\{z \in l_{2}: 2\left(y_{0}-x_{i 0}, z\right) \leq R_{i}^{2}-r^{2}+\left\|y_{0}\right\|^{2}-\left\|x_{i 0}\right\|^{2}\right\}, \quad i \in I_{0} .
\end{aligned}
$$

If $\left(y_{0}-x_{i 0}, p_{0}\right) \geq 0, \quad i \in I$, for a nonzero vector $p_{0}$, and

$$
H\left(y_{0}, r\right) \subset \bigcup_{i \in I} H\left(x_{i 0}, R_{i}\right),
$$

then,

$$
H\left(y_{0}, r\right) \subset \bigcup_{i \in I_{0}} X_{i}
$$


Lemma 3. (See Ibragimov 2005. Assertion 5). Let $\inf _{i \in I} R_{i}=R_{0}>0$ and $\left(y_{0}-x_{i 0}, p_{0}\right) \geq 0, i \in I$, for a nonzero vector $p_{0}$, if for any $0<\delta<R_{0}$ the set $\bigcup_{i \in I} H\left(x_{i 0}, R_{i}-\delta\right)$ does not contain the ball $H\left(y_{0}, r\right)$, then, there exists a point $\bar{y} \in H\left(y_{0}, r\right)$ such that $\left\|\bar{y}-x_{i 0}\right\| \geq R_{i}$, for all $i \in I$.

Theorem 1. Let $\left(y_{0}-x_{i 0}, p_{0}\right) \geq 0$ for all $i \in I$, for a nonzero vector $p_{0} \in l_{2}$, then, the number

$$
\lambda=\inf \left\{l \geq 0: H\left(y_{0}, a(\theta) \sigma\right) \subset \bigcup_{i \in I}^{\infty} H\left(x_{i 0}, a(\theta) \rho_{i}+l\right)\right\}
$$

is the value of the game (2).

Proof. The proof of the theorem in divided into three parts:

1. Constructing the strategies of the pursuers:

We introduce the fictitious pursuers (FPs) $z_{i}$, whose equations of motions are described by

$$
\dot{z}_{i}=\eta(t) w_{i}(t), \quad z_{i}(0)=x_{i 0}, \quad\left(\int_{0}^{\theta}\left\|w_{i}(s)\right\|^{2} d s\right)^{1 / 2} \leq \bar{\rho}_{i}=\rho_{i}+\frac{\lambda}{a(\theta)} .
$$

It can be shown easily that the attainability domain of the fictitious pursuers $z_{i}$ at time $\theta$ from the initial position $x_{i 0}$ is the ball

$$
H\left(x_{i 0}, \bar{\rho}_{i} a(\theta)\right)=H\left(x_{i 0}, a(\theta) \rho_{i}+\lambda\right) .
$$

Next, we define the strategy of the fictitious pursuers $z_{i}, i \in I$, as follows:

$$
w_{i}(t, v)= \begin{cases}\frac{\eta(t)}{a^{2}(\theta)}\left(y_{0}-x_{i 0}\right)+v(t), & \text { if } \quad 0 \leq t \leq \theta_{i 0} \\ 0, & \text { if } \quad \theta_{i 0}<t \leq \theta\end{cases}
$$

where $\theta_{i 0} \in[0, \theta]$ is the time for which

$$
\int_{0}^{\theta_{i 0}}\left\|\frac{\eta(t)}{a^{2}(\theta)}\left(y_{0}-x_{i 0}\right)+v(t)\right\|^{2} d t=\bar{\rho}_{i}^{2}
$$

Note that such time $\theta_{i 0}$ may not exist.

We now define the strategies of the real pursuers $x_{i}, i \in I$, by

$$
u_{i}(t, v)=\frac{\rho}{\bar{\rho}_{i}} w_{i}(t, v), \quad 0 \leq t \leq \theta .
$$

where $\bar{\rho}_{i}=\bar{\rho}_{i}(0)=\rho_{i}+\frac{\lambda}{a(\theta)}$.

2. The value $\lambda$ is guaranteed for the pursuers.

We now show that the strategies (12) satisfy the inequality

$$
\sup _{v(\cdot)} \inf _{i \in I}\left\|y(\theta)-x_{i}(\theta)\right\| \leq \lambda
$$

Thus, it follows from definition of $\lambda$ that

$$
H\left(y_{0}, a(\theta) \sigma\right) \subset \bigcup_{i \in I} H\left(x_{i 0}, a(\theta) \rho_{i}+\lambda\right)
$$

Then, it follows from Lemma 2 where $R_{i}=a(\theta) \rho_{i}+\lambda$ and $r=a(\theta) \sigma$, that

$$
H\left(y_{0}, a(\theta) \sigma\right) \subset \bigcup_{i \in I} X_{i}
$$


where

$$
\begin{aligned}
& I_{0}=\left\{i \in I: S\left(y_{0}, a(\theta) \sigma\right) \cap H\left(x_{i 0}, a(\theta) \rho_{i}+\lambda\right) \neq \varnothing\right\} \\
& \text { and } \\
& X_{i}=\left\{z \in l_{2}: 2\left(y_{0}-x_{i 0}, z\right) \leq\left(a(\theta) \rho_{i}+\lambda\right)^{2}-a^{2}(\theta) \sigma^{2}+\left\|y_{0}\right\|^{2}-\left\|x_{0}\right\|^{2}\right\} .
\end{aligned}
$$

Accordingly, the point $y(\theta) \in H\left(y_{0}, a(\theta) \sigma\right)$ belongs to some half-space $X_{s}$, with $s \in I_{0}$ and hence,

$$
2\left(y_{0}-x_{i s}, y(\theta)\right) \leq\left(a(\theta) \rho_{s}+\lambda\right)^{2}-a(\theta) \sigma^{2}+\left\|y_{0}\right\|^{2}-\left\|x_{s 0}\right\|^{2} .
$$

By Lemma 1, for the strategies of the pursuers $z_{i}, i \in I$, we obtain $z_{s}(\theta)=y(\theta)$ and

$$
\int_{0}^{\theta}\left\|\frac{\eta(t)}{a^{2}(\theta)}\left(y_{0}-x_{s 0}\right)+v(t)\right\|^{2} d t \leq \bar{\rho}^{2} .
$$

By strategy (12), we have

$$
\begin{aligned}
\left\|x_{s}(\theta)-y(\theta)\right\| & =\left\|x_{s}(\theta)-z(\theta)\right\| \\
& =\left\|\int_{0}^{\theta} \eta(t) u_{s}(t) d t-\int_{0}^{\theta} \eta(t) w_{s}(t) d t\right\| \\
& =\left\|\int_{0}^{\theta} \eta(t)\left(\frac{\rho_{s}}{\bar{\rho}_{s}}-1\right) w_{s}(t) d t\right\| \\
& \leq \frac{\lambda}{a(\theta) \bar{\rho}_{s}} \int_{0}^{\theta} \eta(t)\left\|w_{s}(t)\right\| d t .
\end{aligned}
$$

Using Cauchy-Schwatz inequality, we obtain

$$
\int_{0}^{\theta} \eta(t)\left\|w_{s}(t)\right\| d t \leq\left(\int_{0}^{\theta} \eta^{2}(t) d t\right)^{1 / 2}\left(\int_{0}^{\theta}\left\|w_{s}(t)\right\|^{2} d t\right)^{1 / 2} \leq a(\theta) \bar{\rho}_{s} .
$$

Therefore, from inequalities (17) and (18) we obtain

$$
\left\|x_{s}(\theta)-y(\theta)\right\| \leq \lambda
$$

Hence, the value $\lambda$ is guaranteed by the actual pursuers.

3. The value $\lambda$ is guaranteed for the evader.

Define the evader's strategy that satisfies

$$
\inf _{u_{1}(\cdot), \ldots, u_{m}(\cdot), \ldots i \in I} \inf _{i \in I}\left\|y(\theta)-x_{i}(\theta)\right\| \geq \lambda,
$$

where $u_{1}(\cdot), \ldots, u_{m}(\cdot), \ldots$ are the admissible control of the pursuers. If $\lambda=0$, then, the result follows from (20). Suppose that $\lambda>0$, then, by definition of $\lambda$, for any $0<\delta<\lambda$, the set

$$
\bigcup_{i \in I} H\left(x_{i 0}, a(\theta) \rho_{i}+\lambda-\delta\right)
$$

does not include the ball $H\left(y_{0}, a(\theta) \sigma\right)$. According to Lemma 3, there exists a point $\bar{y} \in S(y, a(\theta) \sigma)$ such that $\left\|\bar{y}-x_{i 0}\right\| \geq a(\theta) \rho_{i}+\lambda, i \in I$. Therefore, by the inequality

$$
\left\|x_{i}(\theta)-x_{i 0}\right\| \leq a(\theta) \rho_{i}, \quad i \in I,
$$


We obtain

$$
\left\|\bar{y}-x_{i}(\theta)\right\| \geq\left\|\bar{y}-x_{i 0}\right\|-\left\|x_{i}(\theta)-x_{i 0}\right\| \geq a(\theta) \rho_{i}+\lambda-a(\theta) \rho_{i}=\lambda
$$

Hence, if the evader comes to the point $\bar{y}$ at the time $\theta$, the inequality (20) is guaranteed. This is achievable using the control function

$$
v(t)=\frac{\sigma}{a(\theta)} \eta(t) e, \quad 0 \leq t \leq \theta, \quad e=\frac{\bar{y}-y_{0}}{\left\|\bar{y}-y_{0}\right\|}
$$

that is,

$$
y(\theta)=y_{0}+\int_{0}^{\theta} \eta(t) v(s) d s=\bar{y} .
$$

Hence, the value of the game is not less than $\lambda$ and the inequality is satisfied. This complete the proof of Theorem 1.

We now present some examples to demonstrate our result.

Example 1. Consider the differential game problem (2) with $\rho_{i}=1, \quad \theta=9, \quad \sigma=5$, and denoting $\eta(t)=1$. Consider the following initial positions of the players:

$$
x_{i 0}=(0, \cdots, 0,8,0, \cdots), \quad y_{0}=(0,0, \cdots) .
$$

We can easily see that by simple computation we obtain that $a(\theta)=3, a(\theta) \rho_{i}=3$, and $a(\theta) \sigma=15$.

Next, we show that $\lambda=14$. is value of the game. Firstly, it is enough to show that

i) Given any $\epsilon>0$, the insertion

$$
H(O, 15) \subset \bigcup_{i=1}^{\infty} H\left(x_{i 0}, 17+\epsilon\right)
$$

holds, where $O$ is the origin.

ii) Since the ball $H(O, 15)$ is not contained in the set $\bigcup_{i=1}^{\infty} H\left(x_{i 0}, 13\right)$, that is, let $z=\left(z_{1}, z_{2}, \ldots\right)$ be an arbitrary point of the ball $H(O, 10)$. This implies that $\sum_{i=1}^{\infty} z_{i}^{2} \leq 225$. The following two cases are possible, either $z$ has a non negative coordinate or all coordinates of $z$ are negative. Suppose that there exists a non-negative coordinate $z_{k}$ of the vector $z$, then,

$$
\begin{aligned}
\left\|z-x_{k 0}\right\| & =\left(z_{i}^{2}+\cdots+z_{k-1}^{2}+\left(8-z_{k}\right)^{2}+z_{k+1}^{2}+\ldots\right)^{1 / 2} \\
& =\left(\sum_{i=1}^{\infty} z_{i}^{2}+64-16 z_{k}\right)^{1 / 2} \\
& \leq\left(289-16 z_{k}\right)^{1 / 2} \leq 17 \leq 17+\epsilon
\end{aligned}
$$

hence, $z \in H\left(x_{i 0}, 17+\epsilon\right)$.

Now, suppose that all the coordinates of $z$ are negative. This implies that the inequality

$$
\left\|z-x_{k 0}\right\|=\left(\sum_{i=1}^{\infty} z_{i}^{2}+64-16 z_{k}\right)^{1 / 2} \leq\left(289-16 z_{k}\right)^{1 / 2} \leq 17+\epsilon
$$


is satisfied for sufficiently large $k$, since the series $\sum_{i=1}^{\infty} z_{k^{\prime}}^{2}, z_{k} \rightarrow 0$ as $k \rightarrow \infty$ is convergent. Additionally, any point $z \in S(O, 15)$ with negative coordinates does not belong to the set $\bigcup_{i=1}^{\infty} H\left(x_{i 0}, 13\right)$, since for any number $i,\left\|z-x_{i 0}\right\|=\left(289-16 z_{i}\right)^{1 / 2}>13$.

Therefore, the number

$$
\begin{aligned}
\lambda & =\inf \left\{l \geq 0: \quad H\left(y_{0}, a(\theta) \sigma\right) \subset \bigcup_{i=1}^{\infty} H\left(x_{i 0}, a(\theta) \rho_{i}\right)+l\right\} \\
& =\inf \left\{l \geq 0: \quad H(0,15) \subset \bigcup_{i=1}^{\infty} H\left(x_{i 0}, 3\right)+l\right\}=14
\end{aligned}
$$

is the value of the game by the theorem.

\section{Conclusions}

We have studied a fixed duration pursuit-evasion differential game of a countable number of pursuers pursuing one evader with integral constraints on control functions of the players. We obtained a sufficient condition for completion of pursuit and obtained the value of the game. It worth noting the following points:

- $\quad$ There is no relationship between the players energy resources;

- $\quad$ The case $\eta(t)=1$ was studied in [12].

Author Contributions: I.A., P.K. and G.I. designed the problem. I.A. compiled the manuscript with support from J.R. and W.K. which was concurrently supervised by P.K. and G.I. Finally all authors read and approved the final manuscript.

Funding: Petchra Pra Jom Klao Doctoral Scholarship for Ph.D. program of King Mongkut's University of Technology Thonburi (KMUTT). Theoretical and Computational Science (TaCS) Center. Rajamangala University of Technology Thanyaburi (RMUTTT) (Grant No. NSF62D0604).

Acknowledgments: This project was supported by the Rajamangala University of Technology Thanyaburi (RMUTTT) (Grant No. NSF62D0604). The first author would like to thank King Mongkut's University of Technology Thonburi (KMUTT) for funding this work through Petchra Pra Jom Klao Doctoral Scholarship Academic (Grant No. 13/2561) and 55th Anniversary Commemorative Fund for Ph.D. Program. Furthermore, Poom Kumam was supported by Thailand Research Fund (TRF) and the King Mongkut's University of Technology Thonburi (KMUTT) under TRF Research Scholar Award (Grant No. RSA6080047). Moreover, Wiyada Kumam was financial supported by the Rajamangala University of Technology Thanyaburi (RMUTTT) (Grant No. NSF62D0604) and Ibragimov G.I was supported by Geran Putra Berimpak UPM/700-2/GPD/2017/9590200 of University Putra Malaysia.

Conflicts of Interest: The authors declare no conflict of interest.

\section{References}

1. Isaacs, R. Differential Games. In A Mathematical Theory with Applications to Warfare and Pursuit, Control and Optimization; Courier Corporation: North Chelmsford, MA, USA, 1999.

2. Berkovitz, L.D. A Survey of Differential Games, Mathematical Theory of Control; Academic Press: New York, NY, USA, 1967; pp. 373-385.

3. Friedman, A. Differential Games; John Wiley and Sons: New York, NY, USA, 1971.

4. Krasovskii, N.N. The Theory of Motion Control; Nauka: Moscow, Russia, 1968; Volume 8.

5. Pontryagin, L. Collected Works; Nauka: Moscow, Russia, 1988.

6. Rikhsiev, B. The Differential Games with Simple Motions; Fan: Tashkent, Uzbekistan, 2008.

7. Satimov, N.; Rikhsiev, B. Methods of Solving of Evasion Problems in Mathematical Control Theory; Fan: Tashkent, Uzbekistan, 2000. (In Russian)

8. Subbotin, A.; Chentsov, A. Optimization of Guaranteed Result in Control Problems; Nauka: Moscow, Russia, 1981; Volume 5. 
9. Ibragimov, G.I. Optimal Pursuit with Countably Many Pursuers and one Evader. Differ. Equations 2005, 41, 627-635. [CrossRef]

10. Ibragimov, G.I.; Kuchkarov, A.S. Fixed Duration Pursuit-Evasion Differential Game with Integral Constraints. Math. Phys. Their Appl. 2013, 435. [CrossRef]

11. Ibragimov, G.I.; Abd Rasid, N.; Kuchkarov, A.; Ismail, F. Multi Pursuer Differential Game of Optimal Approach with Integral Constraints on Controls of Players. Taiwan J. Math. 2015, LXXIV, 963-976. [CrossRef]

12. Salimi, M.; Ferrara, M. Differential game of Optimal Pursuit of one Evader by Many Pursuers. Int. J. Game Theory 2018, 13, 481-490. [CrossRef]

13. Salimi, M.; Ibragimov, G.I.; Siegmond, S.; Sharifi, S. On a fixed Duration Pursuit Differential Game with Geometric and Integral Constraint. Dyn. Games Appl. 2016, 30, 409-425. [CrossRef]

14. Azimov, A.; Samatov, B. П-Strategy. An Elementary Introduction to the Theory of Differential Games; NUU Press: Tashkent, Uzbekistan, 2000.

15. Ibragimov, G.I. Collective Pursuit with Integral Constraints on the Controls of the Players. Serberian Adv. Math. 2004, 14, 14-26.

16. Ibragimov, G.; Azamov, A.; Khakestari, M. Solution of a Linear Pursuit-Evasion Game with Integral Constraints. AZIAM J. 2011, 52, 59-75. [CrossRef]

17. Ibragimov, G.I.; Massimiliano, F.; Atamurat, K.; Antonio, P. Simple Motion Evasion Differential Game of many Pursuers and Evaders with integral constraints. Dyn. Games Appl. 2018, 8, 352-378. [CrossRef]

18. Ibragimov, G.I.; Salimi, M.; Amini, M. Evasion from many pursuers in simple motion differential game with integral constraints. Eur. J. Oper. Res. 2012, 218, 505-511. [CrossRef]

19. Leong, W.J.; Ibragimov, G.I. A multiperson pursuit problem on a closed convex set in Hilbert space. Far East J. Appl. Math. 2008 33, 205-214.

20. Idham, A.A.; Ibragimov, G.I.; Kuchkarov, A.S.; Akmal, S. Differential game with many pursuers when controls are subjected to coordinate-wise integral constraints. Malays. J. Math. Sci. 2016, 10, 195-207

21. Levchenko, A.; Pashkov, A. A Game of Optimal Pursuit of one Non-inertial Object by Two Inertial Objects. Prikl. Mekhan. 1985, 8, 49, 536-547.

22. Ibragimov, G.; Satimov, N. A Multiplayer Pursuit Differential Game on a Closed Convex Set with Integral Constraints. Abstr. Appl. Anal. 2012, 2013, 460171. [CrossRef]

(C) 2019 by the authors. Licensee MDPI, Basel, Switzerland. This article is an open access article distributed under the terms and conditions of the Creative Commons Attribution (CC BY) license (http://creativecommons.org/licenses/by/4.0/). 